\title{
Papillary Thyroid Cancer Presenting as a Uterine Metastasis
}

\author{
Christine Newman ${ }^{\mathrm{a}, \mathrm{e}}$, Michael O'Leary ${ }^{\mathrm{b}}$, Denis Quill ${ }^{\mathrm{c}}$, Sine Phelan ${ }^{\mathrm{d}}$, \\ Aoife Lowery ${ }^{\mathrm{c}}$, Derek T. O'Keeffe ${ }^{\mathrm{a}}$, Marcia Bella
}

\begin{abstract}
The objective of this case is to report the highly unusual occurrence of uterine metastasis from a papillary thyroid cancer (PTC). PTC is rarely associated with distant metastases and typically spreads to bone or pulmonary tissue. In this case a 69 -year-old female presented with post menopausal bleeding (PMB). She was otherwise well with no significant medical history or regular medications and reported no family history of thyroid disease. A subsequent endometrial polyp was identified as the cause of her PMB and removed. In addition as part of her clinical examination a goitre was noted and radiological imaging (including an ultrasound, magnetic resonance imaging of the neck and computer tomography of thorax, abdomen and pelvis) was performed and demonstrated a large thyroid mass which extended retrosternally and caused tracheal narrowing. The remainder of the imaging was unremarkable. The endometrial biopsy demonstrated morphological features of PTC. BRAF V600 mutation was not detected. A subsequent core biopsy of the mediastinal mass displayed morphological and immunohistochemical characteristic similar to that of the endometrial polyp. In combination this confirmed a diagnosis of primary papillary thyroid carcinoma. Surgical treatment included a total thyroidectomy, sternotomy and left neck dissection. Lymph node involvement was demonstrated at levels II-VI. Despite radioactive iodine and the use of tyrosine kinase inhibitors this lady died from complications of her disease.
\end{abstract}

Keywords: Papillary thyroid cancer; Metastases; Treatment

\section{Introduction}

Papillary thyroid cancer (PTC) is the most common form of

Manuscript submitted July 18, 2019, accepted August 3, 2019

aDepartment of Endocrinology, Diabetes and Metabolism, Galway University Hospital, Galway, Ireland

bDepartment of Obstetrics and Gynaecology, Galway University Hospital, Galway, Ireland

'Department of Surgery, Galway University Hospital, Galway, Ireland ${ }^{\mathrm{d}}$ Department of Pathology, Galway University Hospital, Galway, Ireland ${ }^{e}$ Corresponding Author: Christine Newman, Department of Endocrinology, Diabetes and Metabolism, Galway University Hospital, Galway, Ireland. Email: Christinenewman89@gmail.com

doi: https://doi.org/10.14740/jem591 thyroid cancer and the incidence is increasing globally. Despite this, PTC rarely metastasises outside of the neck and normally carries an excellent prognosis with 5 -year survival approaching $100 \%$. We describe a highly unusual case of PTC presenting as a uterine metastasis. Ultimately this proved to be an aggressive form of PTC and displayed progression and radioactive iodine (RAI) resistance. Only three additional cases of PTC and uterine metastasis had been described in the literature.

\section{Case Report}

A 69-year-old female presented to the gynecology service with a short history of post menopausal bleeding. She was otherwise well with no significant past medical or family history. During the course of routine physical examination goitre was noted.

\section{Investigation}

An endometrial biopsy was performed to investigate the cause of post menopausal bleeding (PMB). Histology revealed strong thyroid transcription factor-1 (TTF-1) expression. Pax 8 was positive and thyroglobulin was negative. Surprisingly the morphology demonstrated features of PTC with nuclear clearing and overlap and with a rare intranuclear inclusion (Fig. 1a, b). The BRAF V600 mutation (a marker with high negative predictive value for PTC) was not detected. This case presented a diagnostic challenge as TTF 1 expression is seen in uterine tumors, particularly those of mesonephric type; however the morphology was extremely suggestive of PTC.

Given this unusual morphology the patient went on to have an ultrasound of her thyroid, computed tomography (CT) thorax and magnetic resonance imaging (MRI) of neck. These investigations demonstrated a large $10 \times 6 \times 5 \mathrm{~cm}$ heterogeneous, complex, partially solid-cystic mass in the left anterior triangle replacing the left hemithyroid. The right side of thyroid appeared normal. The mass extended retrosternally to the level of the arch of the aorta and displaced the trachea towards the right, causing approximately $50 \%$ stenosis. There were multiple subcentimeter bilateral cervical lymph nodes present. The mediastinum was otherwise normal with no suspicious pulmonary nodules. The liver, kidneys, spleen, pancreas and adrenal glands were normal. There was no evidence of retroperitoneal, 

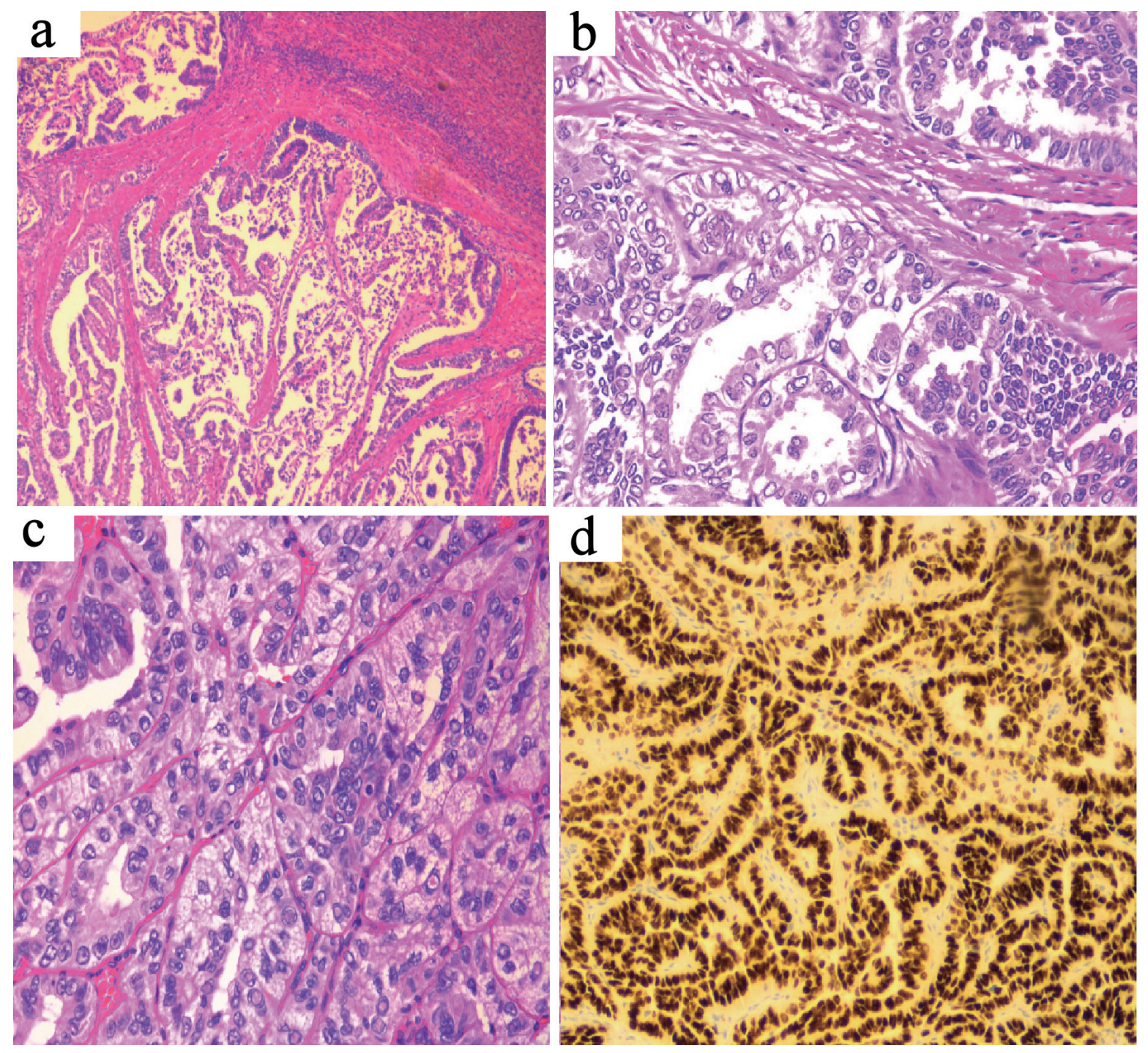

Figure 1. (a) Uterine tumor (H\&E stain, $\times 10$ magnification) showing tumor deposit within myometrium. (b) Uterine tumor (H\&E stain, $\times 20$ magnification) showing typical morphology of papillary thyroid carcinoma, morphologically similar to the patient's thyroid tumor. (c) Thyroid tumor (H\&E stain, $\times 20$ magnification) showing typical papillary thyroid carcinoma with prominent intranuclear inclusions. (d) TTF-1 immunostain of thyroid tumor, $\times 20$ magnification, showing strong diffuse expression.

mesenteric or pelvic lymphadenopathy. There were no destructive bone lesions and a bone scan was normal.

A mediastinal core biopsy was performed and the results showed tubulo-papillary and tubular growth pattern. The nuclei show cytoplasmic clearing and overlap. Intranuclear cytoplasmic inclusions are present. The specimen stained positive for cytokeratin 7 (CK7) and TTF-1, however monoclonal anti-carcinoembryonic antigen (mCEA) was negative (Fig. 1c, d). The histology was identical to the endometrial samples taken; confirming a diagnosis of PTC with uterine metastasis.

\section{Treatment}

Surgical treatment consisted of a total thyroidectomy, sternoto- my and left neck dissection. Surgical specimens demonstrated PTC, with focal "tall cell" features and lymphovascular invasion. Lymph node involvement was demonstrated at level (II, III, IV) (2/14 nodes) and level (V, VI) (1/22 nodes). Thymic tissue was unremarkable. The final grade was $\mathrm{pT} 3 \mathrm{~N} 1 \mathrm{~b}$.

A total abdominal hysterectomy and bilateral salpingooophorectomy was performed 8 weeks later and histology confirmed that the two lesions were cytolomorphologically identical (Fig. 1).

Following RAI ablation of 7,400 Mbq, a post treatment scan showed some residual uptake in the left side of the thyroid bed and in the midline superior to the expected position of the thyroid. There were no visible iodine-avid metastases and patient was clinically well. The patient continued on regular surveillance and received a suppressive dose of levothyroxine to aim for a thyroid stimulating hormone (TSH) level of $<0.1$ 
$\mathrm{mIU} / \mathrm{L}$.

\section{Outcome and follow-up}

Unfortunately 9 months later, this lady represented with PMB caused by a vaginal polyp. Imaging revealed a vaginal vault lesion, left adnexal mass and right paratracheal lymph node. Histology again confirmed metastatic PTC. Her treatment was discussed by a multi-disciplinary team and the patient received external beam radiotherapy and RAI at a dose of $5,500 \mathrm{GBq}$ with a satisfactory post-treatment scan undertaken at $72 \mathrm{~h}$.

Three months later the patient complained of shoulder pain at a routine follow-up visit. Despite a normal examination this prompted a full restaging and repeat imaging showed intra-abdominal, thoracic and lymph node disease. One of the liver lesions was biopsied and confirmed papillary thyroid malignancy. As the post-treatment RAI uptake scan did not demonstrate any of the lesion visualised on CT imaging it was determined that the disease was non-RAI avid and alternative therapies should be considered.

Under the guidance of the oncology team, lenvatinib (a tyrosine kinase inhibitor (TKI)) was commenced. A followup scan 6 months later showed the burden of omental disease had reduced however the patient ultimately passed away from complications of pulmonary and abdominal metastases.

\section{Discussion}

PTC is the most common form of thyroid cancer and typically bears an excellent prognosis with 5-year survival of nearly $100 \%$ [1]. Despite frequent local lymph node involvement, only $13.3 \%$ present with distant metastases [2].

Negative predictive factors of reduced overall survival include tall cell morphology [2]; macroscopic lymph node metastasis which predicts very high risk for persistence postsurgery [3]; age greater than 60 years, radioiodine refractory disease and positron emission tomography (PET) avidity [4], size of thyroid lesion $(>4 \mathrm{~cm})$ and male gender [1].

The presence of distant metastases also has a significant effect on long-term survival and reduces 5-year survival from nearly $100 \%$ to $56 \%$ [5]. Lung and bone metastases are commonly seen in PTC and account for $75 \%$ and $17 \%$ of metastases respectively [5].

Additional published case reports have identified other atypical sites for metastases from PTC including pituitary metastases and lesions in the occipital skull and heart.

To date only three additional cases of uterine metastases from PTC has been reported in the literature [6-8]. In this instance the patient had a reassuring post-operative RAI scan which did not demonstrate abnormal uptake and at the time of publishing the patient was clinically well.

Uterine metastases typically occur in women with a previous malignancy and as such this case represents a true clinical rarity.

Treatment options for aggressive metastatic or recurrent PTC (outside of thyroid surgery, RAI and suppressive doses of thyroid hormone replacement) are limited by both burden of disease and toxicity of the available treatments. As seen in this case, RAI is the first-line treatment for metastatic disease. For patients displaying satisfactory responses following RAI therapy the overall survival at 10 years is $92 \%$, versus $19 \%$ in those who display persistent disease [9]. Despite the greatly reduced overall survival in the group with persistent disease, outcomes are still preferable to those who display no RAI uptake at all-survival rates of $15 \%$ vs. $8 \%$ respectively [9].

Indications for moving on from RAI to other forms of treatment include disease which becomes refractory to RAI (25-50\% of metastatic PTC) or receiving a cumulative dose of RAI $>22,000 \mathrm{MBq}[10]$.

Currently two tyrosine kinase inhibitors (TKIs) (lenvatinib and sorafenib) have Food and Drug Administration (FDA) approval for the treatment of metastatic PTC. Though showing improvements in progression-free survival and objective response rate, changes in overall survival have been disappointing. A number of other TKIs are at earlier stages of trial and show promising results in terms of progression-free survival. There is also evidence to suggest some additional benefit from the use of alternative TKI therapy when one has been unsuccessful [10].

Despite promising benefits the side effects of TKI therapies are significant and include cardiac, hepatic, cutaneous and renal complications. These medications are also teratogenic, myelosuppressive and cause elevations in TSH. As such a comprehensive evaluation of pre-existing symptoms and thorough counselling of patients are advised before initiation of therapy.

As our ability to obtain molecular profiles of thyroid cancer causing mutations becomes more sophisticated, other therapies also become available. Such examples include vemurafenib and dabrafenib, selective inhibitors of the BRAF mutation which is present in $51 \%$ of PTC. In small studies, use of these agents produced a partial response rate of over $30 \%$, and despite being associated with significant side effects, they are potentially future therapeutic options [11]. Additional small molecules which have been studied in clinical trials with limited numbers include MEK inhibitors which serve to cause re-sensitisation of thyroid tumor to RAI, phosphoinositide 3-kinase (PIK3) and tropomyosin-related kinase (TRK) inhibition.

Systemic chemotherapy can be considered in RAI refractory patients in whom other therapies (including TKIs) are unsuccessful or unsuitable. At present there are too few data to recommend any specific regimen.

Finally the most recent development for treatment of metastatic PTC to show promise is peptide receptor radionuclide therapy (PRRT); a method of targeting somatostatin receptors which has been used successfully in the treatment of neuroendocrine tumors. Though very few studies using PRRT have shown overall survival benefit in PTC, this area shows significant therapeutic promise and warrants further study [12].

\section{Conclusions}

We present the case of a highly unusual pattern of metastatic 
spread in the setting of PTC. This displayed an aggressive phenotype, and ultimately displayed RAI and TKI resistance. More recent developments in the treatment of PTC were unavailable to the patient at the time of disease presentation and progression.

\section{Acknowledgments}

None to declare.

\section{Financial Disclosure}

None to declare.

\section{Conflict of Interest}

The authors declare that there is no conflict of interest that could be perceived as prejudicing the impartiality of the research reported.

\section{Informed Consent}

Informed consent was obtained from the family of this patient.

\section{Author Contributions}

$\mathrm{CN}$ and DTO'K contributed equally to the writing of the case report. SP, DQ, AL, MO'L all contributed to patient's case and MB was the physician in charge of the patient's care and edited the case report.

\section{References}

1. Ito Y, Miyauchi A, Kihara M, Fukushima M, Higashiyama T, Miya A. Overall survival of papillary thyroid carcinoma patients: a single-institution long-term follow-up of 5897 patients. World J Surg. 2018;42(3):615-622.

2. Vischal Rao US, Koya S, Chatterjee S, Patil AA, Nayar RC, Kallur KG. Evaluation of distant metastasis in treated thyroid cancers: Study of Radioiodine scans in a tertiary care center. Journal of Head and Neck Physicians and Surgeons. 2016;4(2):59-62.

3. de Castro TP, Waissmann W, Simoes TC, de Mello RC, Carvalho DP. Predictors for papillary thyroid cancer persistence and recurrence: a retrospective analysis with a 10-year follow-up cohort study. Clin Endocrinol (Oxf). 2016;85(3):466-474.

4. Schmidt A, Iglesias L, Klain M, Pitoia F, Schlumberger MJ. Radioactive iodine-refractory differentiated thyroid cancer: an uncommon but challenging situation. Arch Endocrinol Metab. 2017;61(1):81-89.

5. Nixon IJ, Whitcher MM, Palmer FL, Tuttle RM, Shaha AR, Shah JP, Patel SG, et al. The impact of distant metastases at presentation on prognosis in patients with differentiated carcinoma of the thyroid gland. Thyroid. 2012;22(9):884-889.

6. Wang JH, Yu J, Ning CP, Sun YM, Fang SB. Papillary thyroid carcinoma with massive metastasis in the uterine corpus: a case report. BMC Cancer. 2013;13:551.

7. Kumar NB, Hart WR. Metastases to the uterine corpus from extragenital cancers. A clinicopathologic study of 63 cases. Cancer. 1982;50(10):2163-2169.

8. Benbassat CA, Mechlis-Frish S, Hirsch D. Clinicopathological characteristics and long-term outcome in patients with distant metastases from differentiated thyroid cancer. World J Surg. 2006;30(6):1088-1095.

9. Durante C, Haddy N, Baudin E, Leboulleux S, Hartl D, Travagli JP, Caillou B, et al. Long-term outcome of 444 patients with distant metastases from papillary and follicular thyroid carcinoma: benefits and limits of radioiodine therapy. J Clin Endocrinol Metab. 2006;91(8):28922899.

10. Schlumberger M, Tahara M, Wirth LJ, Robinson B, Brose MS, Elisei R, Habra MA, et al. Lenvatinib versus placebo in radioiodine-refractory thyroid cancer. N Engl J Med. 2015;372(7):621-630.

11. Flaherty K, Puzanov I, Sosman J, Kim K, Ribas A, McArthur G, Lee RJ, et al. Phase 1 study of PLX4032: Proof of concept for V600E BRAF mutation as a therapeutic target in human cancer. J Clin Oncol (Meeting Abstracts). 2009;27:90000.

12. Faugeras L, Pirons AS, Donchier J, Michel L, Lemaure J, Vandervost S, D'Hondt L. Refractory thyroid carcinoma: which systemic treatment to use. Therapeutic Advances in Medical Oncology. 2018;10. 